\title{
Attitude towards pediatric resuscitation among health care professional in an Italian hospital.
}

Enzo Picconi, Luca Tortorolo, Orazio Genovese, Marco Piastra, Giorgio Conti

Pediatric Intensive Care Unit, Department of Intensive Care Medicine and Anesthesiology, Fondazione Policlinico Universitario A. Gemelli - Università Cattolica del Sacro Cuore, Rome,

Gemelli :

Fondazione Policlinico Universitario A. Gemelli niversità Cattolica del Sacro Cuore Italy

Objective. To describe the attitudes of health care professionals working in an Italian hospital (Fondazione Policlinico Universitario "A. Gemelli" in Rome) towards cardiopulmonary resuscitation (CPR) on infants and children.

Materials and methods. A selfadministered survey question-naire, comprising five questions, was distributed to professionals who were willing to undergo the European Pediatric Advance Life Support Course (EPALS). The questions were related to discomfort and attitudes to pediatric CPR, assessing by the use of a 10-point Likert scale. Data about answers are expressed as mean and standard deviation (SD) or as a percentage, as appropriated.

Results. All 42 health care professionals (9.5\% anesthesiologists, $\quad 7.1 \%$ pediatricians, $11.9 \%$ residents in anesthesia and intensive care, $54.8 \%$ residents in pediatrics, $11.9 \%$ nurses, $4.8 \%$ "others" $=$ other physicians,

physiotherapists; mean age 32.4 years; $73.8 \%$ female) completed the questionnaire. $76.2 \%$ had an experience over one year in pediatric field and 59.5\% had already taken part to a basic pediatric life support course. In the event of a child who needs CPR, most participants admit that they would feel anxious (mean 7.0, SD 2.1), that they would feel uncertain about their abilities

(mean 6.0, SD 2.0) and that they would leave room for others (mean 6.1, SD 2.6). From the examination of the answers, a certain fear to damage children (mean 5.3, SD 2.6) and to use the defibrillator (mean 5.7, SD 2.8) emerge.

Conclusions. Cardiac arrest is a rare event in pediatric age and most professionals showed little attitude towards pediatric CPR. On the basis of this information, our institution developed an educational planning, according to which all health practitioners involved in the care of pediatric patients attend the EPALS course. This program intends to optimize confidence in the performance of pediatric CPR, and potentially to save lives.

If you had to manage a child who needs cardiopulmonary resuscitation:
How much would you be afraid to harm the baby?
How much would you be afraid to use the defibrillator?
Would the situation make you anxious?
Would you be sure of your skills?
Would you intervene or leave room for others?

Figure 1. Summary of confidence in performing pediatric resuscitation. 